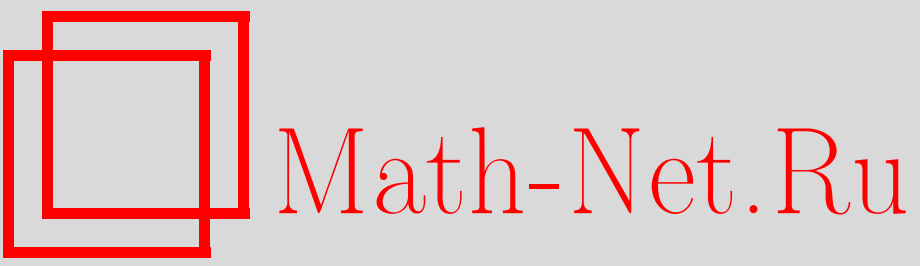

Д. В. Ширков, К поведению эффективной связи КХД в инфракрасной области, ТМФ, 2002, том 132, номер 3, 484-496

DOI: https://doi.org/10.4213/tmf376

Использование Общероссийского математического портала Math-Net.Ru подразумевает, что вы прочитали и согласны с пользовательским соглашением

http://www.mathnet.ru/rus/agreement

Параметры загрузки:

IP: 18.208 .226 .222

26 апреля 2023 г., 15:20:25 
ТЕОРЕТИЧЕСКАЯ

И МАТЕМАТИЧЕСКАЯ

ФИЗИКА

Том 132, № 3

сентябрь, 2002

(C) 2002 г.

\author{
Д.В. Ширков*
}

\title{
К ПОВЕДЕНИЮ ЭФФЕКТИВНОЙ СВЯЗИ КХД В ИНФРАКРАСНОЙ ОБЛАСТИ
}

Обзор непертурбативных результатов для эффективной функции связи $\mathrm{KX} Д \bar{\alpha}_{s}$, получаемых методами численных симуляций континуального интеграла на решетке и решения приближенных уравнений Швингера-Дайсона, обнаруживает замечательное разнообразие поведения $\bar{\alpha}_{s}\left(Q^{2}\right)$ в инфракрасной области, даже на качественном уровне. В свою очередь, это поднимает вопрос о соответствии результатов, находимых различными группами авторов. Эта ситуация рассматривается с привлечением зависящих от массовой переменной преобразований константы связи, сделан вывод о том, что задача инфракрасного поведения эффективной функции связи, а также пропагаторов, в КХД покамест не является достаточно хорошо определенной и ее постановка требует дальнейшего уточнения.

Ключевые слова: квантовая хромодинамика, континуальный интеграл, непертурбативная инфракрасная асимптотика.

\section{1. ВВЕДЕНИЕ}

Это исследование посвящено вопросу поведения, а также определения инвариантной функции связи (ИФС) квантовой хромодинамики (КХД) $\bar{\alpha}_{s}$ в инфракрасной (ИК) области. Понятие ИФС является центральным в современной практике квантово-полевых применений метода ренормализационной группы (РГ). В КТП само наличие РГ тесно связано с конечными перенормировочными преобразованиями Дайсона. Поэтому ИФС, или инвариантный заряд, определяется (см. пионерские работы [1]-[3], а также главу "Ренормализационная группа" в монографиии [4]) через произведение, включающее перенормировочные факторы $z_{i}$, которое инвариантно по отношению к указанным преобразованиям, без какого-либо использования предела слабой связи или ультрафиолетовой (УФ) асимптотики.

Выражая факторы $z_{i}$ через значения перенормированных функций КТП, обычно получают выражение для ИФС $\bar{\alpha}\left(Q^{2}\right)$ как функции одного аргумента кинематического типа - переданного импульса $Q \equiv \sqrt{Q^{2}=\mathbf{Q}^{2}-Q_{0}^{2}}$ (либо импульса отсчета) - в виде произведения константы связи, амплитуд пропагаторов и некоторой вершинной функции в

* Лаборатория теоретической физики им. Н.Н. Боголюбова, Объединенный институт ядерных исследований, Дубна, Московская обл., Россия. E-mail: shirkovd@thsun1.jinr.ru 
импульсном представлении (см. ниже уравнения (1)-(3)). При этом все перенормированные величины соотнесены с какой-либо определенной схемой перенормировки.

Подобные определения были введены и использованы в контексте теории возмущений еще в середине 50-х годов. В современной практике $\bar{\alpha}\left(Q^{2}\right)$ обычно рассматривается в УФ-пределе. Так назьваемая безмассовая перенормировочная схема минимальных вычитаний $\overline{\mathrm{MS}}$ оказывается наиболее удобной и потому наиболее употребительной в УФ-анализе.

Между тем упомянутые определения справедливы для более общего, содержащего зависимость от масс(ы), случая (см. [3]-[5]). В калибровочных моделях КТП, содержаших несколько вершин с одной и той же константой связи, эта массовая зависимость, в свою очередь, приводит к специфической вериинной зависимости, т.е. зависимости от вида вершинной функции, использованной в определении ИФС. Например, в КХД могут быть использованы трехглюонная, глюон-духовая, четырехглюонная и различные глюон-кварковые вершины. В безмассовой $\overline{\mathrm{MS}}$-схеме все ИФС, построенные на основе различных вершин, являются одинаковыми. Однако в массивном случае разница между ними может оказаться сушественной в области $Q \lesssim m[6]$.

Здесь уместно прокомментировать различие между безмассовыми и "массивными" схемами вычитаний и возникающими на их основе РГ-решениями. Так, например, сушествуют два варианта $\overline{\mathrm{MS}}$-схемы. Ограничимся моделью КТП с одной константой связи $g$. В обшепринятом варианте, для модели КТП с одной константой связи $g$, на основе безмассовых контрчленов строится бета-функция одного аргумента $\beta(g)$. Решение дифференциального РГ-уравнения для ИФС (и других функций) приводит к выражению $\bar{g}=f(\ell, g)$, зависяшему от логарифма $\ell=\ln \left(Q^{2} / \mu^{2}\right)$ и описываюшему УФ-асимптотику. В то же время в более обшем формализме РГ-генераторы (бета- и гамма-функции), определяемые на основе перенормированных в $\overline{\mathrm{MS}}$-схеме, зависящих от массовой переменной $m$ исходных приближенных выражений, оказываются функциями двух безразмерных переменных $Q^{2} / m^{2}$ и $g$. Решения РГ-уравнений приводит к выражениям, зависящим от дополнительного массового аргумента $m^{2} / \mu^{2}$. Для ИФС общее выражение имеет вид $\bar{g}\left(Q^{2} / \mu^{2}, m^{2} / \mu^{2}, g\right)$. Массовая зависимость становится сушественной в ИК-области. Таким образом в КЭД была описана в свое время ИК-особенность пропагатора электрона [2], [7].

Указанная массовая зависимость, по нашему мнению, проявляется в современном непертурбативном анализе свойств КХД в ИК-области, в частности в вычислениях функционального интеграла на решетке и в решениях усеченных уравнений Швингера-Дайсона (УУШД). Различные группы исследователей берут за основу разные определения перенормированной константы связи и ИФС в ИК-области. Например, тюбингенская группа использует [8] для определения ИФС глюон-духовую вершину, парижская группа [9], [10] - трехглюонную, а “трансокеанская" группа [11], [12] - глюон-кварковую.

Раздел 2 содержит краткий обзор результатов для $\bar{\alpha}_{s}$, полученных различными группами авторов путем решеточных симулящий и решений УУШД, приводящих к примечательно пестрой картине качественно разнообразных поведений в ИК-области.

В разделе 3 мы напоминаем происхождение понятия ИФС и отмечаем, что оно не свя- 
зано с теорией возмушений и УФ-асимптотикой. Рассмотрены вопрос о неоднозначности определения $\bar{\alpha}_{s}\left(Q^{2}\right)$ и формализм преобразований константы связи и сопутствующих преобразований ИФС. При обсуждении используются преобразования константы связи, подобные известным преобразованиям между различными схемами перенормировок, содержашие зависимость от масс(ы). Здесь оказывается сушественным понятие вериинной зависимости.

В разделе 4 мы возврашаемся к вопросу о соответствии между различными ИК-асимптотиками $\bar{\alpha}_{s}\left(Q^{2}\right)$. В частности, построены модельные примеры преобразований ИФС, сингулярных в ИК-области и в отдельных случаях приводящих к ИК-асимптотикам, качественно близким к приведенным в разделе 2 .

Наш вывод состоит в том, что проблема ИК-асимптотик эффективной связи $\bar{\alpha}_{s}\left(Q^{2}\right)$ (а также пропагаторов) не является сформулированной достаточно четко. Результаты различных групп, полученные в импульсном представлении, не следует сравнивать между собой напрямую. Одним из возможных критериев для сравнения остаются результаты расчетов адронных характеристик.

\section{2. НЕПЕРТУРБАТИВНЫЕ РЕЗУЛЬТАТЫ В ИК-ОБЛАСТИ}

Этот раздел содержит компендиум непертурбативных результатов, полученных различными группами при вычислениях ИФС $\bar{\alpha}_{s}\left(Q^{2}\right)$ в ИК-области путем численных симуляций на решетке и с помошью приближенных решений УУШД. Каждая группа использует свое собственное определение для $\bar{\alpha}_{s}\left(Q^{2}\right)$, совместимое с режимом асимптотической свободы в УФ-области, и получает собственные, отличные от других, результаты в ИК-области.

2.1. Тюбингенская группа: глюоны и духи. Исследования группы из Тюбингена (см. обзор в работе [8]) ведутся параллельно по двум направлениям: решение УУШДи решеточные симуляции четырехмерного евклидова континуального интеграла для квантовой глюодинамики. Таким образом, рассматривается лишь глюон-духовый сектор КХД, что оказывается особенно удобным для построения и анализа УУШД в поперечной калибровке. Здесь перенормировочная функция Г глюон-духовой вершины в силу тождеств Уорда-Славнова представима через соответствуюшие функции для глюонного $Z$ и духового $G$ пропагаторов. В то же время в калибровке Ландау функция $\Gamma$ выпадает из определения ИФС, построенной на глюон-духовой вершине:

$$
\bar{\alpha}_{\mathrm{T}}\left(Q^{2}\right)=\alpha_{s} Z\left(Q^{2}\right) G^{2}\left(Q^{2}\right) .
$$

Поэтому только два пропагатора входят в усеченные надлежашим образом уравнения Швингера-Дайсона.

Симуляции на решетке для пропагаторов показывают [13], [14] весьма специфическое ИК-поведение. Глюонная функция перенормировки $Z\left(Q^{2}\right)$ (она же поперечная амплитуда глюонного пропагатора) при убывании переданного импульса проходит через максимум при $Q=\sqrt{Q^{2}} \simeq 1$ ГэВ и затем спадает к нулю примерно как $Q$, амплитуда духового пропагатора $G\left(Q^{2}\right)$ монотонно возрастает и имеет степенную ИК-сингулярность, 
близкую к $Q^{-1 / 2}$, в то время как их произведение $(1)$ для $\bar{\alpha}_{\mathrm{T}}\left(Q^{2}\right)$ монотонно стремится к конечному ИК-пределу. Эта картина качественно подтверждается результатами решения УУШД, которые приводят [13]-[15] к степенным ИК-асимптотикам

$$
G\left(Q^{2}\right) \simeq(Q)^{-\kappa}, \quad Z\left(Q^{2}\right) \simeq\left(Q^{2}\right)^{\kappa}, \quad \kappa=0.595,
$$

и предельному ИК-значению $\bar{\alpha}_{\mathrm{T}}(0)=2.97=8.92 / N_{c}$.

2.2. Парижская группа: трехглюонная вершина. В работах парижской группы ИФС строится на основе несимметрично вычтенной трехглюонной вершины $\widetilde{\Gamma}\left(Q^{2}\right) \equiv$ $\Gamma_{3 \mathrm{gl}}\left(Q^{2}, 0, Q^{2}\right)$ в калибровке Ландау для модели КХД с двумя массивными кварками. В этой схеме перенормировки ИФС

$$
\bar{\alpha}_{\mathrm{P}}\left(Q^{2}\right)=\alpha_{s} \widetilde{\Gamma}^{2}\left(Q^{2}\right) Z^{3}\left(Q^{2}\right)
$$

согласно выгислениям на решетке имеет весьма своеобразное поведение в ИК-области [9]. При убывании $Q$ она проходит через максимум при $Q \simeq 1-1.5$ ГэВ, а затем резко падает и оказьвается близкой к нулю при $Q \simeq 0.5$ ГэВ. Количества данных и их точности не хватает для определения ИК-асимптотики, однако не исключено степенное убывание вида $\sim\left(Q^{2}\right)^{\nu}, \nu \gtrsim 2$.

2.3. "Трансокеанская" группа: глюон-кварковая вершина. "Трансокеанская" группа ${ }^{1)}$ использует вычисления на решетке для КХД с двумя массивными кварками. Рассчитана эффективная функция связи, определенная на основе кварк-глюонной вершины в схеме импульсных вычитаний при нулевом моменте глюона $\Gamma_{\mathrm{TO}}\left(Q^{2}\right) \equiv \Gamma_{\mathrm{q}-\mathrm{gl}}\left(0 ; Q^{2}, Q^{2}\right)$. Результаты для ИФС

$$
\bar{g}_{\mathrm{TO}}\left(Q^{2}\right)=g \Gamma_{\mathrm{TO}}\left(Q^{2}\right) Z^{1 / 2}\left(Q^{2}\right) S\left(Q^{2}\right)
$$

( $S$ - амплитуда кваркового пропагатора), полученные путем решеточных симулящий, качественно близки (см. рис. 4 в работе [11] или рис. 8 и 9 в работе [12]) к результатам вычислений парижской группы. Однако ИК-асимптотика, по-видимому, имеет другой показатель

$$
\bar{g}(Q) \simeq\left(Q^{2}\right)^{\mu / 2}, \quad \bar{\alpha} \simeq\left(Q^{2}\right)^{\mu}, \quad \mu \lesssim 1 .
$$

2.4. Группа "ALPHA": функционал Шредингера. Группа "ALPHA" изучает КХД с двумя безмассовыми кварковыми ароматами и использует для решеточных симуляций так называемый функционал Шредингера, определенный на четырехмерном евклидовом пространственно-временном многообразии специальным образом: по трем пространственным измерениям наложены циклические граничные условия, тогда как "временная" координата выделена - значения калибровочных полей на "верхней" и "нижней" крышках отличаются на фазовый фактор $e^{-\eta}$. Перенормированная

\footnotetext{
1) Название условно, поскольку эта группа включает исследователей из удаленных друг от друга центров, в частности из Австралии, Великобритании и континентальной Европы.
} 
эффективная функция связи $\bar{\alpha}_{\mathrm{SF}}$ определена через производную $\Gamma^{\prime}=\partial \Gamma / \partial \eta$ эффективного действия, представленного в виде разложения

$$
\Gamma=\alpha^{-1} \Gamma_{0}+\Gamma_{1}+\alpha \Gamma_{2}+\cdots
$$

как функция пространственного размера $L$ многообразия (см. уравнения $(2.43)$ в работе [16] и (8.3) в [17])

$$
\bar{\alpha}_{\mathrm{SF}}(L)=\frac{\Gamma_{0}^{\prime}}{\Gamma^{\prime}}
$$

т.е. как функция в координатном представлении. Для изучения эволюции $\bar{\alpha}_{\mathrm{SF}}(L)$ использован особый прием [17]. Вводится ступенчатая функиия подобия

$$
\sigma\left(\bar{\alpha}_{\mathrm{SF}}(L)\right)=\bar{\alpha}_{\mathrm{SF}}(2 L),
$$

для явного нахождения которой следует знать бета-функцию. В то же время функция $\sigma$ может быть определена численно из сравнения результатов решеточных симуляций для решеток размером $L$ и $2 L$ (см. рис. 16 в работе [17]). Полученные на этой основе недавние результаты [18] выявляют чрезвычайно быстрый рост $\bar{\alpha}_{\mathrm{SF}}(L)$ в области $L \rightarrow \infty$ при $\bar{\alpha}_{\mathrm{SF}} \simeq 1$. Аналитическая аппроксимация для результатов численных расчетов поведения $\bar{\alpha}_{\mathrm{SF}}$ имеет экспоненциальную (!) форму

$$
\bar{\alpha}_{\mathrm{SF}}(L) \simeq e^{M L}, \quad M \simeq \frac{2.3}{L_{\max }},
$$

где $L_{\max }$ - точка отсчета в области достаточно слабой связи - неявно определяется условием $\bar{\alpha}_{\mathrm{SF}}\left(L_{\max }\right)=0.275$, что дает $L_{\max } \sim 1 / m_{\tau}$. При физической интерпретации $\left.\bar{\alpha}_{\mathrm{SF}}(L)\right)$ подразумевается “обычное квантово-механическое" отождествление $L=1 / Q$.

\section{3. ФУНКЦИЯ СВЯЗИ, ЗАВИСЯШАЯ ОТ МАСС(Ы)}

Технически выделяют схемы перенормировок, в которых параметр шкалы $\mu$ совпадает с импульсом вычитания (так называемые МОМ-схемы). Для них условие нормировки ИФС имеет простой вид

$$
\left.\bar{g}\right|_{Q^{2}=\mu^{2}}=g_{\mu} .
$$

В общем случае ${ }^{2)}$, к которому, в частности, относятся схемы минимальных вычитаний, в том числе обшепринятая $\overline{\mathrm{MS}}$-схема, это условие содержит “функцию нормировки”

$$
\left.\bar{g}\right|_{Q^{2}=\mu^{2}}=N(g),
$$

которая может зависеть от масс(ы).

Условие нормировки (6) отвечает наиболее простому функциональному уравнению для ИФС, которое в безмассовом приближении имеет вид

$$
\bar{g}\left(x ; g_{\mu}\right)=\bar{g}\left(\frac{x}{t} ; \bar{g}\left(t ; g_{\mu}\right)\right), \quad x=\frac{Q^{2}}{\mu^{2}} .
$$

При этом условие (6) оказывается следствием уравнения (8). Мы будем называть его каноническим. Для случая (7) функциональное уравнение имеет более сложный вид (см. ниже уравнение (14)), в котором также учтена зависимость от массы $m$.

\footnotetext{
${ }^{2)} \mathrm{B}$ этом разделе для константы связи, наряду с $g$, употребляется обозначение $\alpha=g^{2} /(4 \pi)$, более принятое в калибровочных моделях.
} 
3.1. "Массивный” случай. Перейдем к более общему случаю, когда учитывается зависимость от массы. Здесь ИФС приобретает дополнительный аргумент, который удобно выбрать (см., например, [4]) в виде безразмерного отношения $m^{2} / \mu^{2}=y$. Условие нормировки для ИФС $\bar{g}(x, y ; g)$, обобшаюшее (7), можно записать в виде

$$
\bar{g}_{N}(1, y ; g)=N(y, g),
$$

отличном от канонического. Оно содержит функцию двух переменных.

Отметим, что здесь введено новое специальное обозначение $\bar{g}_{N}$ для ИФС с условием нормировки (9) $N \neq g$. Мы сохраняем прежнее обозначение $\bar{g}$ для частного случая ИФС, нормированной каноническим образом (6). При этом будем использовать термины ИФС для $\bar{g}_{N}$ с условием нормировки (9) и әффективная функция связи (ЭФС) или әффективная связь для $\bar{g}$, нормированной канонически.

Эффективная связь в массивном случае удовлетворяет довольно простому функциональному уравнению

$$
\bar{g}(x, y ; g)=\bar{g}\left(\frac{x}{t}, \frac{y}{t} ; \bar{g}(t, y ; g)\right), \quad x=\frac{Q^{2}}{\mu^{2}}, \quad y=\frac{m^{2}}{\mu^{2}},
$$

а также групповым дифференциальным уравнениям

$$
x \frac{\partial \bar{g}(x, y ; g)}{\partial x}=\beta\left(\frac{y}{x}, \bar{g}\right), \quad \mathcal{X}_{\beta} \cdot \bar{g}(x, y ; g)=0,
$$

где

$$
\mathcal{X}_{\beta} \equiv\left\{x \frac{\partial}{\partial x}+y \frac{\partial}{\partial y}-\beta(y, g) \frac{\partial}{\partial g}\right\},\left.\quad \beta(y, g) \equiv \frac{\partial \bar{g}(\xi, y ; g)}{\partial \xi}\right|_{\xi=1} .
$$

Уравнения (11) и (12) являются обобщениями более известных безмассовых уравнений и переходят в них при $y=0$. Мы даем специальное обозначение $\mathcal{X}_{\beta}$ для инфинитезимального группового оператора (оператора Ли), который в данном контексте был введен Овсянниковым [19] в 1956 г. Его нижний индекс отвечает координате последнего слагаемого. Мы будем называть $\mathcal{X}$ оператором Ли-Овсянникова.

Э $\Phi \mathrm{C} \bar{g}$ по определению удовлетворяет каноническому условию нормировки

$$
\bar{g}(1, y ; g)=g,
$$

совместному с функциональным уравнением (10), тогда как прочие РГ-функции одного аргумента, подобные амплитудам пропагаторов и моментам структурных функций, в МОМ-схеме при $Q^{2}=\mu^{2}$ обычно нормированы на единицу.

Функциональное уравнение для ИФС имеет более сложную форму

$$
\bar{g}_{N}(x, y ; g)=\bar{g}_{N}\left\{\frac{x}{t}, \frac{y}{t} ; n\left[\frac{y}{t}, \bar{g}_{N}(t, y ; g)\right]\right\},
$$

которая отражает групповой закон сложения и отвечает условию нормировки (9). В него входит функция $n$, обратная к $N$ по отношению ко второму аргументу. 
Дифференцируя уравнение (14) двумя различными способами, получаем групповые дифференциальные уравнения

$$
\frac{\partial \bar{g}_{N}(x, y ; g)}{\partial \ln x}=B\left(\frac{y}{x} ; \bar{g}_{N}(x, y ; g)\right), \quad \mathcal{X}_{\beta} \cdot \bar{g}_{N}(x, y ; g)=0
$$

причем

$$
\begin{gathered}
B(y, g)=\left.\frac{\partial}{\partial \xi} \bar{g}_{N}\{\xi, y ; n(y, g)\}\right|_{\xi=1}, \\
\beta(y, g)=\left.\left\{B(y, \gamma) \frac{\partial}{\partial \gamma}-y \frac{\partial}{\partial y}\right\} n(y, \gamma)\right|_{\gamma=N(y, g)} .
\end{gathered}
$$

Итак, в случае ИФС мы имеем дело с двумя различными бета-функииями $B$ и $\beta$, входящими в разные дифференциальные уравнения (15) и связанными между собой вторым из соотношений (16).

Заметим, что ИФС $\bar{g}_{N}$ можно трактовать как ЭФС, отнесенную к другой константе связи $\gamma \equiv N(y, g)$. В самом деле, вводя новую функцию связи

$$
\bar{\gamma}(x, y ; \gamma)=\bar{g}_{N}\{x, y ; n(y, \gamma)\}
$$

находим, что она удовлетворяет простому функциональному уравнению вида (10), дифференциальным уравнениям с оператором Ли-Овсянникова $\mathcal{X}_{B}$ с одним и тем же генератором $B(y, \gamma)$ и может рассматриваться как ЭФС для константы связи $\gamma$.

С другой стороны, если определить некоторую новую функцию $\tilde{g}$ соотношением

$$
\tilde{g}(x, y ; g)=n\left\{\frac{y}{x}, \bar{g}_{N}(x, y ; g)\right\}=n\left\{\frac{y}{x}, \bar{\gamma}(x, y ; N(y, g))\right\},
$$

то она также будет удовлетворять простому функциональному уравнению (10) и может рассматриваться на равных основаниях с ИФС $\bar{\gamma}$. Оба дифференциальных уравнения для $\tilde{g}$ содержат один и тот же генератор $\beta$. Мы назовем эту функцию групповой функцией связи, чтобы отметить, что, вообще говоря, она не связана с ИФС преобразованием Дайсона.

3.2. Преобразования, зависящие от массы. Рассмотрим теперь в общем, зависяшем от массовой переменной, случае преобразование от константы связи $\gamma$ и ИФС $\bar{\gamma} \mathrm{k}$ новой константе связи $g$ и ЭФС $\bar{g}$. К последовательности таких преобразований сводится, в частности, переход от популярной безмассовой $\overline{\mathrm{MS}}$-схемы к упоминавшейся выше массивной $\overline{\mathrm{MS}}$-схеме.

Положим без потери общности, что соответствующие константы связи $\gamma$ и $g$ связаны соотношениями $\gamma=N(y, g), g=n(y, \gamma)$. Тогда согласно (18) ИФС соотносятся как

$$
\bar{\gamma}(x, y ; \gamma)=N\left\{\frac{y}{x}, \bar{g}(x, y ; g)\right\}
$$

а соответствуюшие групповые генераторы связаны соотношениями (16).

Иными словами, переход

$$
g \rightarrow \gamma=N(y, g)
$$


можно рассматривать как преобразование, обусловленное изменением способа перенормировки. Таким образом, от набора $\{g, \bar{g}(x, y ; g), \beta(y, g)\}$ можно перейти к другому набору $\{\gamma, \bar{\gamma}(x, y ; \gamma), B(y, \gamma)\}$ с помошью преобразования (20), а также соотношения (19), представленного в виде

$$
\bar{g} \rightarrow \bar{\gamma}(x, y ; \gamma)=N\left\{\frac{y}{x}, \bar{g}\left[x, y ; n\left(\frac{y}{x}, \gamma\right)\right]\right\}
$$

и являющегося композицией (17) и (18). Генераторы $\beta$ и $B$ связаны формулой (16). При этом в групповых функциональных уравнениях для функций $s(x, y ; g)$ (подобных амплитудам пропагаторов) следует не только учитывать связь (18) между ИФС $\bar{g}_{N}$ и ЭФС $\tilde{g}$

$$
s(x, y ; g)=\frac{s(t, y ; g)}{s\{1, y ; \tilde{g}(t, y ; g)\}} s\left\{\frac{x}{t}, \frac{y}{t} ; \tilde{g}(t, y ; g)\right\}
$$

но также и нормировку самой функции $s$

$$
s(1, y ; g)=S(y, g) \neq 1 .
$$

Соответствующие дифференциальные уравнения имеют вид

$$
\frac{\partial \ln s(x, y ; g)}{\partial \ln x}=\gamma\left[\frac{y}{x}, \tilde{g}(x, y ; g)\right], \quad \mathcal{X}_{\beta} \cdot \ln s(x, y ; g)=\tilde{\gamma}\left(\frac{y}{x}, g\right),
$$

где

$$
\gamma(y, g)=\left.\frac{\partial}{\partial t} \ln s(t, y ; g)\right|_{t=1}, \quad \tilde{\gamma}(y, g)=\gamma(y, g)+\left\{y \frac{\partial}{\partial y}-\beta(y, g) \frac{\partial}{\partial g}\right\} \ln S(y, g) .
$$

В конкретной калибровочной модели КТП как ИФС, так и ЭФС определяются схемой перенормировки, включая выбор вершины и способ ее вычитания.

3.3. Вершинная зависимость. В калибровочных моделях для массивного случая в определении ЭФС возникает специфическая степень свободы, обусловленная наличием в лагранжиане нескольких структур с одной и той же константой связи. Как известно, при введении ИФС используются одетые вершинные функции - так называемые вертексы. Таким образом, появляются различные возможности для определения ИФС в той жсе самой схеме перенормировки. Переход между различными ИФС может быть описан в терминах только что описанных преобразований.

Для иллюстрации возьмем одетые вершины КХД: трехглюонную $\Gamma_{3 \mathrm{gl}}$, глюон-духовую $\Gamma_{\mathrm{gl}-\mathrm{gh}}$, глюон-кварковую $\Gamma_{\mathrm{gl-q}}$, а также четырехглюонную $\Gamma_{4 \mathrm{gl}}$. Вообще говоря, каждая из них может быть использована при определении ИФС. Например,

$$
\bar{\alpha}_{3 \mathrm{gl}}=\alpha_{s} \Gamma_{3 \mathrm{gl}}^{2} Z^{3}, \quad \bar{\alpha}_{\mathrm{gl}-\mathrm{gh}}=\alpha_{s} \Gamma_{\mathrm{gl}-\mathrm{gh}}^{2} Z G^{2}, \quad \bar{\alpha}_{\mathrm{gl}-\mathrm{q}}=\alpha_{s} \Gamma_{\mathrm{gl}-\mathrm{q}}^{2} Z S^{2},
$$

где $Z, G$ и $S$ - скалярные амплитуды глюонного, духового и кваркового пропагаторов.

В безмассовом УФ-пределе все дефиниции могут оказаться близкими: они совпадают, в частности, в $\overline{\mathrm{MS}}$-схеме и лишь слегка отличаются в разных МОМ-схемах из-за 
различных возможностей определения функции $\bar{\alpha}_{i}$, выражаемой через какой-либо из вертексов, представленных в виде функции одного пространственноподобного аргумента $Q^{2}$.

Однако в массивном случае функции $\bar{\alpha}_{i}$ могут значительно разниться в ИК-области из-за различных зависимостей от масс кварков. Кварковые пропагаторы и глюон-кварковые вертексы имеют сингулярности на массовой поверхности. Для легких кварков это приводит к сингулярному ИК-поведению.

Глюон-духовая вершина, а следовательно, и $\bar{\alpha}_{\mathrm{gl}-\mathrm{gh}}$, по всей видимости, должна быть наименее чувствительной по отношению к массовым эффектам. В самом деле, в однопетлевом приближении зависимость от масс кварков входит в $\bar{\alpha}_{\mathrm{gl}-\mathrm{gh}}$ только через вклад петли кварковой поляризации вакуума в амплитуду глюонного пропагатора $Z$. Однако этот вклад не имеет пороговой сингулярности. Две другие конструкции $\bar{\alpha}_{3 \mathrm{gl}}$ и $\bar{\alpha}_{\mathrm{gl}-\mathrm{q}}$ оказываются более чувствительными к ИК-сингулярностям. Массовые эффекты появляются не только через амплитуду кваркового пропагатора $S$, но и через вертексы $\Gamma_{3 g l}$ или $\Gamma_{\mathrm{gl}-\mathrm{q}}$.

Возможно, именно этому отвечает более “спокойный” вариант ИК-поведения $\bar{\alpha}_{\mathrm{T}}\left(Q^{2}\right)$, полученный тюбингенской группой.

\section{4. МОДЕЛЬНЫЕ ПРЕОБРАЗОВАНИЯ КОНСТАНТ СВЯЗИ}

Здесь представлены примеры модельных преобразований констант связи и индуцированных ими преобразований ИФС, в том числе в случае наличия зависимости от массовой переменной. Показано, что подходяшим преобразованием константы связи $\alpha_{s}$ можно сушественно изменить поведение $\bar{\alpha}\left(Q^{2}\right)$ как функции $Q^{2}$ в ИК-области.

4.1. Преобразования из аналитической теории возмущений. Приведемпримеры, навеянные так назьваемой аналитической теорией возмущений (АТВ) - недавно развитой конструкцией [20], [21], позволяющей "бескровным образом" (за счет наложения условия аналитичности Челлена-Лемана) избавлять пертурбативную КХД от нефизических особенностей типа полюса Ландау.

В АТВ переход от общепринятой Э $\Phi \mathrm{C} \bar{\alpha}_{s} \overline{\mathrm{MS}}$-схемы к аналитическим функциям свя-

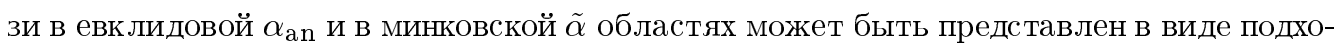
дящего преобразования константы связи $\alpha_{s}$, подобного преобразованию при изменении схемы перенормировки [22]. В однопетлевом случае

$$
\begin{aligned}
\alpha_{s} \rightarrow \alpha_{\mathrm{M}}\left(\alpha_{s}\right) & =\frac{1}{\pi \beta_{0}} \arccos \frac{1}{\sqrt{1+\pi^{2} \beta_{0}^{2} \alpha_{s}^{2}}}=\frac{1}{\pi \beta_{0}} \operatorname{arctg}\left(\pi \beta_{0} \alpha_{s}\right), \\
\alpha_{s} \rightarrow \alpha_{\mathrm{E}}\left(\alpha_{s}\right) & =\alpha_{s}+\frac{1}{\beta_{0}}\left(1-e^{1 /\left(\beta_{0} \alpha_{s}\right)}\right)^{-1}
\end{aligned}
$$

Первое из этих преобразований выглядит вполне обычно ${ }^{3)}$, поскольку $\alpha_{\mathrm{M}}\left(\alpha_{s}\right)$ может быть разложена по степеням $\alpha_{s}$, как и при обычных схемных преобразованиях, тогда как

\footnotetext{
3) Отметим, что подобная функция исходной константы связи $\alpha_{\mathrm{M}}\left(\alpha_{s}\right)$ возникала в точном решении двумерной модели Тирринга [23].
} 
второе в случае слабой связи сводится к тождественному, поскольку непертурбативная структура $\simeq e^{-1 / \beta_{0} \alpha_{s}}$ не "оставляет следов" в степенном разложении.

Если начинать с известной однопетлевой функции $\bar{\alpha}_{s}\left(Q^{2}\right) \sim 1 / \ln \left(Q^{2} / \Lambda^{2}\right)$ с полюсом первой степени при $Q^{2}=\Lambda^{2}$, то в результате подстановки (26) и (27) в групповое дифференциальное уравнение и его интегрирования мы придем к известным в АТВ выражениям (первое из которых в физическом контексте АТВ является функцией $s$, а не $Q^{2}$ )

$$
\begin{gathered}
\tilde{\alpha}\left(Q^{2}\right)=\left.\frac{1}{\pi \beta_{0}} \arccos \frac{L}{\sqrt{L^{2}+\pi^{2}}}\right|_{L>0}=\frac{\operatorname{arctg}(\pi / L)}{\pi \beta_{0}}, \\
\alpha_{\mathrm{E}}\left(Q^{2}\right)=\frac{1}{\beta_{0}}\left[\frac{1}{L}-\frac{\Lambda^{2}}{Q^{2}-\Lambda^{2}}\right] ; \quad L=\ln \frac{Q^{2}}{\Lambda^{2}},
\end{gathered}
$$

Функции преобразования $\alpha_{\mathrm{M}}\left(\alpha_{s}\right)$ и $\alpha_{\mathrm{E}}\left(\alpha_{s}\right)$ обладают важными свойствами:

AC: они стремятся к $\alpha_{s}$ в пределе слабой связи, т.е. $\alpha_{i} \rightarrow \alpha_{s}$ при $\alpha_{s} \ll 1$;

СП: они конечны при $\alpha_{s}=\infty$;

ИКк: они ИК-конечны, т.е. $\alpha_{i} \rightarrow 1 / \beta_{0}$ в пределе $\alpha_{s} \rightarrow-0$.

Условие $\mathbf{A C}$ в случае слабой связи обеспечивает соответствие со свойством асимптотической свободы, свойство СП приводит к отсутствию "призрачных", т.е. нефизических, сингулярностей и, наконец, свойство ИКк отвечает конечному ИК-пределу. Последнему свойству не удовлетворяет преобразование

$$
\alpha_{s} \rightarrow \alpha_{\mathrm{N}}=\alpha_{s}\left(1-e^{-1 /\left(\beta_{0} \alpha_{s}\right)}\right)
$$

эквивалентное полученному в работе [24] в модифицированной АТВ.

В силу свойств $\mathbf{A C , ~} \mathbf{C П ~ и ~ И К к ~ о б е ~ н о в ы е ~ А Т В - и н в а р и а н т н ы е ~ ф у н к ц и и ~ с в я з и ~ ( 2 8 ) ~}$ и $(29)^{4)}$

1) обладают свойством асимптотической свободы;

2) свободны от нефизических сингулярностей при $Q^{2} \simeq \Lambda^{2}$;

3) имеют конечный ИК-предел при $Q^{2}=0$.

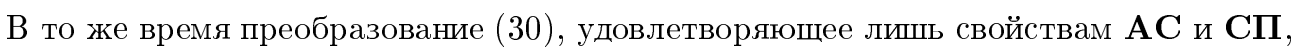
приводит к сингулярному в ИК-пределе выражению для $\bar{\alpha}_{\mathrm{N}}$ :

$$
\bar{\alpha}_{\mathrm{N}}\left(Q^{2}\right)=\frac{Q^{2}-\Lambda^{2}}{\beta_{0} Q^{2} \ln \left(Q^{2} / \Lambda^{2}\right)}
$$

Это выражение обладает дополнительной $Q^{-2}$-особенностью, которая, по мнению некоторых исследователей, отвечает линейному росту межкваркового потенциала с расстоянием.

\footnotetext{
${ }^{4)}$ Бета-функции для обеих однопетлевых функций $\tilde{\alpha}^{(1)}$ и $\alpha_{\text {an }}^{(1)}$ имеют нуль при $\alpha=1 / \beta_{0}$ и симметричны относительно отражения $\left[\alpha-1 /\left(2 \beta_{0}\right)\right] \rightarrow-\left[\alpha-1 /\left(2 \beta_{0}\right)\right]$. Кроме того, бета-функция для $\tilde{\alpha}(s)$ оказывается одновременно спектральной функцией для $\alpha_{\mathrm{an}}\left(Q^{2}\right)$. Последнее свойство, своеобразно реализующее гипотезу Швингера [25], не зависит от однопетлевого приближения [22], [26].
} 
Приведем еще один пример безмассового преобразования

$$
\alpha_{s} \rightarrow \alpha_{\mathrm{SF}}=\alpha_{s} \frac{e^{b}-\exp \left\{b e^{-1 /\left(2 \beta_{0} \alpha_{s}\right)}\right\}}{e^{b}-1}, \quad b=\frac{M}{\Lambda},
$$

приводящего к выражению

$$
\bar{\alpha}_{\mathrm{SF}}\left(Q^{2}\right)=\frac{1}{\beta_{0} \ln \left(Q^{2} / \Lambda^{2}\right)} \frac{e^{b}-e^{b \Lambda / Q}}{e^{b}-1}
$$

с экспоненциальной сингулярностью $\sim e^{M / Q}$ при $Q=0$, которое напоминает аналитическую аппроксимацию (5) для результатов в ИК-области, полученную недавно [18] группой “ALPHA” на основе численных симуляций на решетке для ИФС, определенной для функционала Шредингера. Подобная сушественная особенность противоречит представлению Челлена-Лемана. Отметим, что выражение (32), в свою очередь, обладает существенной сингулярностью по переменной $\alpha_{s}$ при $\alpha_{s}=0$.

Из приведенных примеров следует простое правило соответствия между преобразованиями констант связи типа (26), (27) и индуцированными ими выражениями (28), (29) для ИФС:

при безмассовом преобразовании константы связи $\alpha_{s} \rightarrow \alpha_{i}=f_{i}\left(\alpha_{s}\right)$ соответствующая ИФС преобразуется по правилу

$$
\bar{\alpha}_{s}\left(Q^{2}\right) \rightarrow \bar{\alpha}_{i}\left(Q^{2}\right)=f_{i}\left(\bar{\alpha}_{s}\left(Q^{2}\right)\right)
$$

4.2. Примеры массивного преобразования. Обратимся теперь к преобразованиям константы связи, зависящим от массовой переменной. В общем случае согласно (20) такое преобразование включает функцию двух переменных

$$
\alpha_{s} \rightarrow \alpha^{*}=N\left(y, \alpha_{s}\right)
$$

При этом преобразование ЭФС выглядит следующим образом:

$$
\bar{\alpha}_{s} \rightarrow \bar{\alpha}^{*}\left(x, y ; \alpha^{*}\right)=N\left\{\frac{y}{x}, \bar{\alpha}_{s}\left[x, y ; n\left(\frac{y}{x}, \alpha_{s}\right)\right]\right\}
$$

где, в отличие от (34), содержится переменная $y / x=m^{2} / Q^{2}$, которая также может существенно повлиять на поведение в ИК-области.

Для иллюстрации сказанного рассмотрим модельное выражение

$$
N(y, \alpha)=\alpha_{\mathrm{M}}\left(\alpha_{s}\right)\left(\frac{1-y}{1+y}\right)^{c \alpha_{\mathrm{M}}\left(\alpha_{s}\right)}
$$

которому отвечает функция

$$
\bar{\alpha}^{*}\left(Q^{2}\right)=\tilde{\alpha}\left(Q^{2}\right)\left(\frac{Q^{2}-m^{2}}{Q^{2}+m^{2}}\right)^{c \tilde{\alpha}\left(Q^{2}\right)}
$$


Входящие сюда константа связи $\alpha_{\mathrm{M}}\left(\alpha_{s}\right)$ и ЭФС $\tilde{\alpha}\left(Q^{2}\right)$ определены формулами вида $(26)$ и (28). При этом мы можем отвлечься от однопетлевого приближения. Здесь сушественно лишь то, что функция $\tilde{\alpha}\left(Q^{2}\right)$ свободна от нефизических сингулярностей и монотоно возрастает к ИК-пределу, где принимает конечное значение $\tilde{\alpha}(0) \simeq 1.4$, не зависяшее от петлевого приближения. Структура введенной сингулярности напоминает известную ИК-особенность (см. [4]) вертекса КЭД на массовой поверхности.

При положительном индексе $c \simeq 1$ и небольшой массе кварка $m \ll \Lambda$ функция $\bar{\alpha}^{*}\left(Q^{2}\right)$, определенная выражением (38), может довольно успешно имитировать описанные в п. 2.2 и 2.3 результаты численных симуляций $\bar{\alpha}_{s}$, полученные парижской и “трансокеанской" группами.

\section{5. ОБСУЖДЕНИЕ}

Как было показано в разделе 2 , результаты по непертурбативной КХД, полученные различными группами путем решеточных вычислений евклидова континуального интеграла, а также приближенных решений уравнений Швингера-Дайсона, сушественно различаются в ИК-области, в особенности для ЭФС $\bar{\alpha}_{s}$. В то же время "физические" результаты этих групп по адронным характеристикам кажутся более согласованными между собой.

Наши "модельные конструкции" для $\bar{\alpha}_{s}$ (см. раздел 4 ) показывают, что ИК-поведение эффективной связи сушественно зависит от деталей ее определения. При конструировании мы ограничились классом моделей, которые в УФ-области коррелируют с пертурбативной КХД. Такое соответствие обычно считается достаточным при решеточных симуляциях и анализе решений уравнений Швингера-Дайсона.

На практике именно такие "допустимые" $\bar{\alpha}_{i}$, удовлетворяюшие условию $\mathbf{A C}$, могут соответствовать различным решеточным выгислениям. Расчеты в рамках подобных схем качественно описывают конфайнмент и некоторые адронные характеристики. Поэтому схемы с допустимыми $\bar{\alpha}_{i}$ можно рассматривать как "физические".

Особый интерес привлекает ЭФС, полученная группой "ALPHA", с ее экспоненциальным ростом $\sim e^{M L}(5)$ при $L \rightarrow \infty$. Здесь при переходе от координатного к импульсному представлению требуется особая осторожность ввиду нарушения условия применимости теоремы Таубера при преобразовании Фурье. Этот вопрос более подробно будет рассмотрен в другом месте.

В итоге, по нашему мнению, можно заключить, что нет какого-либо смысла в попытках определения "истинного" ИК-поведения пертурбативной ЭФС КХ Д $\bar{\alpha}_{s}$. Любая инфракрасная КХД-физика, подобная физике адронов или физике $\tau$-распада, не может быть успешно описана лишь в рамках пертурбативной КХД. Для ее разумного описания нужны либо сушественно непертурбативные методы, такие, например, как вычисления функционального интеграла, либо паллиативные схемы [27]-[29], дополняющие пертурбативную КХД феноменологическими параметрами, наподобие "эффективных партонных масс" или аномальных вакуумных средних.

Благодарности. Автор выражает признательность Р. Алкоферу, Г. Алтарелли, Б. А. Арбузову, М. Люшеру, И. Л. Соловцову и Р. Стора за полезные обсуждения и за- 
мечания. Эта работа была начата в Теоретическом отделе ЦЕРН, руководству которого автор благодарен за гостеприимство. Она была частично поддержана грантами РФФИ № 00-15-96691 и № 02-01-00601, а также INTAS № 96-0842 и INTAS-CERN № 99-0377.

\section{Список литературы}

[1] Н. Н. Боголюбов, Д. В. Ширков. ДАН СССР. 1955. Т. 103. С. 203.

[2] Н. Н. Боголюбов, Д. В. Ширков. ДАН СССР. 1955. Т. 103. С. 391.

[3] N. N. Bogoluibov, D. V. Shirkov. Nuovo Cimento. 1956. V. 3. P. 845.

[4] Н. Н. Боголюбов, Д. В. Ширков. Введение в теорию квантованных полей. М.: Наука, 1984.

[5] D. V. Shirkov. Nucl. Phys. B. 1992. V. 371. P. 467.

[6] Yu.L. Dokshitzer, D. V. Shirkov. Z. Phys. C. 1995. V. 67. P. 449.

[7] А.А. Логунов. ЖЭТФ. 1956. Т. 30. С. 793.

[8] L. Alkofer, L. von Smekal. Phys. Rep. 2001. V. 353. P. 281; hep-ph/0007355.

[9] Ph. Boucaud et al. Nucl. Phys. B (Proc. Suppl.). 2002. V. 106. P. 266; hep-ph/0110171.

[10] Ph. Boucaud et al. JHEP. 2002. V. 0201. P. 046; hep-ph/0107278.

[11] J. I. Skullerud, A. Kizilersu, A. G. Williams. Nucl. Phys. B (Proc. Suppl.). 2002. V. 106. P. 841; hep-lat/0109027.

[12] J. Skullerud, A. Kizilersu. Quark-gluon vertex from lattice QCD. hep-th/0205318.

[13] C. S. Fischer, L. Alkofer. Phys. Lett. B. 2002 . V. 536. P. 177; hep-ph/0202202.

[14] C.S. Fischer, R. Alkofer, H. Reinhardt. Phys. Rev. D. 2002. V. 65. P. 094008; hep-ph/0202195.

[15] Ch. Lerche, L. von Smekal. Phys. Rev. D. 2002. V. 65. P. 125006.

[16] M. Lüscher et al. Nucl. Phys. B. 1992. V. 384. P. 168.

[17] M. Lüscher. Advanced lattice QCD. In: Probing the Standard Model of Particle Interactions. Eds. R. Gupta et al. Amsterdam: Elsevier Sc, 1999. P. 229.

[18] J. Heitger, H. Simma, R. Sommer, U. Wollf. Nucl. Phys. B. (Proc. Suppl.). 2002. V. 106. P. 859; hep-lat/0110201.

[19] Л. В. Овсянников. ДАН СССР. 1956. Т. 109. С. 1112.

[20] D. V. Shirkov, I. L. Solovtsov. Phys. Rev. Lett. 1997. V. 79. P. 1209; hep-ph/9704333.

[21] D. V. Shirkov, I. L. Solovtsov. Phys. Lett. B. 1998. V. 442. P. 344; hep-ph/9711251.

[22] D. V. Shirkov. Eur. Phys. J. 2001. V. 22. P. 331.

[23] W. E. Thirring. Ann. Phys. 1958. V. 3. № 1. P. 91-112.

[24] A. V. Nesterenko. Phys. Rev. D. 2000. V. 62. P. 094028.

[25] J. Schwinger. Proc. Nat. Acad. Sci. USA. 1974. V. 71. P. 3024; P. 5047.

[26] Д. В. Ширков. ТМФ. 2001. Т. 127. С. 3; hep-ph/0012283.

[27] F. Jegerlehner, A. V. Tarasov. Nucl. Phys. B. 1999. V. 549. P. 481.

[28] K. A. Milton, I. L. Solovtsov, O. P. Solovtsova. Phys. Rev. D. 2001. V. 64. P. 016005; 2002. V. 65. P. 076009.

[29] M. Baldicci, G. M. Prosperi. Infrared behaviour of the running coupling and bound states in QCD. hep-ph/0202172. 